\title{
Capabilités et adaptation en Île-de-France. De la difficulté à intégrer les capabilités dans les plans locaux d'adaptation au changement climatique
}

\author{
Mélanie Pommerieux ${ }^{1, *}$, Nathalie Blanc ${ }^{2}$ (D) Lydie Laigle ${ }^{3}$ et Stéphane Tonnelat ${ }^{4}$ \\ 1 Science Politique, CNRS, UMR LADYSS, Paris, France \\ 2 Géographie, CNRS, UMR LADYSS, Paris, France \\ ${ }^{3}$ Sociologie, CSTB, Université Paris Est, Marne-la-Vallée, France \\ ${ }^{4}$ Anthropologie, CNRS, UMR LAVUE, Paris, France
}

Reçu le 7 janvier 2019. Accepté le 14 février 2020

Si l'adaptation est devenue un concept incontournable pour penser le rapport entre l'environnement et la société, le défi aujourd'hui est certainement sa mise en pratique sur le terrain, « [...] étape plus délicate au regard de son histoire étymologique complexe et duale qui s'entremêle avec d'autres concepts [...]» comme le signalait Guillaume Simonet dans la revue en 2009 (NSS, 17, 4, 392-401). C'est ce qui est proposé ici, avec un focus sur plusieurs territoires franciliens où des plans d'adaptation locaux sont appelés à être opérationnels. La capacité transformationnelle de ces adaptations est ainsi examinée au prisme de leur aptitude à modifier les orientations initiales en fonction des contraintes décelées. Au sein de ce processus, la capabilité mobilisable chez le citoyen apparaît comme un enjeu déterminant pour aller vers une mise en œuvre locale plus effective.

La Rédaction

Résumé - Alors que l'attention portée à l'échelle mondiale à l'adaptation au changement climatique s'intensifie, l'objet de cet article est d'étudier l'évolution des stratégies d'adaptation à l'échelle locale, au sein de trois territoires franciliens. Notamment parce que l'incertitude liée aux risques est forte au niveau local, nous plaidons pour une approche transformationnelle de l'adaptation, s'appuyant sur les capabilités. Nous montrons toutefois que l'adaptation reste principalement envisagée sous l'angle de la réduction des risques environnementaux. Jusqu'à présent, la participation du public met peu en avant les capabilités valorisées par les populations. La désectorialisation des politiques climatiques permet néanmoins de mieux inclure les citoyens.

Mots-clés : capabilités / adaptation / changement climatique / participation publique / niveau local

\begin{abstract}
Capabilities and adaptation in Île-de-France: the difficult integration of capabilities into local climate change adaptation schemes. Multilateral agreements at the global level have not succeeded in stabilizing the concentration of greenhouse gases in the atmosphere. Following this assessment, a new way to look at climate issues seems necessary. Interest in adaptation is growing internationally, and transformational adaptation emerges today as a promising concept to address strategies of both mitigation and adaptation. In this article, we propose a new approach to consider local adaptation strategies based on capabilities. Relying on three case studies of local authorities in Île-deFrance, we characterize their recent local adaptation strategies and analyse how these have taken capabilities into account. Different institutional tools allow for various ways of integrating capabilities. Plans Climat, which rely on technical studies, address administrations and professionals rather than citizens. Agendas 21 may provide a more holistic approach, but fail to address citizens' needs in order for them to act for
\end{abstract}

\footnotetext{
*Auteur correspondant : melanie.pommerieux@sciencespo.fr
} 
adaptation. As for calls for associative or citizens projects, competition for funding does not foster cooperation. We demonstrate that the limitations put on public participation processes do not yet allow for the emergence and reinforcement of capabilities. Most of the local strategies studied in fact correspond to an adjustment approach to adaptation. However, spurred by recent objectives to strongly reduce emissions, new ways of integrating citizens' contributions into public policy have emerged.

Keywords: capabilities / adaptation / climate change / public participation / local level

« Penser autrement la question climatique ${ }^{1} »$ (Aubertin et al., 2015) implique de réfléchir aux complémentarités entre l'atténuation et l'adaptation au changement climatique.

L'adaptation transformationnelle se distingue de l'adaptation qualifiée tour à tour de résilience, d'ajustement, incrémentale, ou de gestion des risques, qu'elle soit anticipatrice ou réactive. Si l'approche identifiant l'adaptation à un ajustement entend répondre à la question "s'ajuster à quoi », en identifiant les risques climatiques actuels ou anticipés, puis les vulnérabilités, l'adaptation transformationnelle propose un pas de côté. Elle permet d'interroger le comment, «adapter quoi à quoi », afin non plus d'ajuster les territoires aux besoins et pratiques anthropiques, mais de transformer ces pratiques pour les «faire correspondre à ce que peut offrir le territoire » (Simonet, 2016). Penser l'adaptation en ces termes est désormais une nécessité. D'abord, l'inéluctabilité du changement climatique ne fait plus question, et ses effets sont d'ores et déjà perceptibles (Marquet et Salles, 2014). Il s'agit alors de réfléchir à des stratégies d'adaptation et d'atténuation qui font sens conjointement. Ensuite, commencer par définir des risques ne fait guère sens dans la mesure où l'essentiel des changements climatiques est silencieux (Simonet, 2016) et où la période est et restera marquée par l'incertitude au niveau local (Godard, 2010). Comme le souligne le Groupe d'experts intergouvernemental sur l'évolution du climat (GIEC) depuis 2014², l'adaptation est fortement dépendante du contexte, mais aussi des valeurs et des priorités des populations. C'est pourquoi nous définissons l'adaptation transformationnelle comme « $\mathrm{s}$ 'appuyant sur l'interaction entre les systèmes sociaux et les changements environnementaux de long terme » (Blanc et Laigle, 2018).

\footnotetext{
${ }^{1}$ Le numéro supplément de Natures Sciences Sociétés publié en 2015 proposait de réunir des contributions étant à la fois force de réflexion et de propositions, au vu des limites des accords multilatéraux relatifs au changement climatique depuis 23 ans (https://www.nss-journal.org/fr/articles/nss/ abs/2015/02/contents/contents.html).

2 Simonet (2015) démontre notamment qu'à partir de 2014, l'adaptation devient la «colonne vertébrale » d'un des rapports du GIEC, en reconnaissant que la prise en compte du changement climatique, à toutes les échelles d'organisation, est désormais inéluctable.
}

Dans le cadre du projet de recherche CAPADAPT ${ }^{3}$, nous avançons que l'expression conceptuelle de capabilités telle que développée par Amartya Sen (1999) et Martha Nussbaum (2000) permet de faire le lien entre les réflexions sur l'adaptation transformationnelle et le développement de capacités inédites de prise en charge citoyenne de problèmes locaux, notamment en relation avec des problèmes plus globaux.

Dans cet article, nous proposons d'étudier des plans d'adaptation au niveau local en Île-de-France afin d'analyser la manière dont les capabilités y sont intégrées en faisant le lien avec la définition de l'adaptation retenue. Les communes françaises ont-elles fait évoluer leurs stratégies d'adaptation pour aller vers une approche transformationnelle? Quelle place ont-elles accordée aux capabilités jusqu'alors? Qui a pu y contribuer?

Les recherches sur les politiques climatiques locales soulignent le manque de dialogue entre acteurs politicoadministratifs locaux et citoyens à propos de la définition des objectifs de réduction des émissions de gaz à effet de serre, «très souvent définis dans une logique mimétique et a-territoriale » (Brédif et al., 2015). Cette focale sur les émissions conduirait à une impasse: les politiques climatiques manquent de légitimité, car elles ont «souvent négligé la question des modes de vie et de leurs évolutions» (Aubertin et al., 2015). Plus qu'un problème de couplage entre science et politique, elles pâtiraient donc au contraire d'une trop forte approche technique, associée à une faible mise en dialogue entre citoyens et politiques.

Dans le cadre des plans d'adaptation locaux, nous émettons l'hypothèse que l'absence de prise en compte des capabilités citoyennes, définies ici sommairement

\footnotetext{
${ }^{3}$ Cet article s'inscrit dans le cadre du projet CAPADAPT (2017/2020), adaptation des villes au changement climatique et «capabilités» : vers une approche en termes de développement humain. Ce projet est financé dans le cadre du programme Gestion et impact du changement climatique (GICC) par l'Agence de l'environnement et de la maîtrise de l'énergie (ADEME). Une première phase de recherche, dont cet article est issu, a abouti à la rédaction d'une synthèse destinée à l'ADEME, intitulée "Analyse des évolutions récentes des trajectoires d'adaptation au changement climatique dans trois collectivités d'Île-de-France ».
} 
comme «les capacités des citoyens à mobiliser leurs expériences et relations aux milieux, en vue d'enrichir leurs opportunités d'être et d'agir en prenant conscience des différents facteurs qui affectent leurs conditions de vie » (Sen, 2010), empêche la définition de stratégies d'adaptation transformationnelle, qui proposeraient des changements de pratiques plutôt qu'un ajustement des territoires aux besoins anthropiques.

Après une présentation de notre cadre théorique puis de notre méthodologie, nous analyserons la place accordée aux capabilités dans les stratégies d'adaptation locales en fonction de différents types d'outils institutionnels de planification.

\section{Opérationnaliser le lien entre adaptation et capabilités via la participation publique et l'empowerment}

Plusieurs études ont proposé de faire le lien entre capabilités et adaptation. Toutefois, à notre sens, elles n'ont pas encore pris la mesure des évolutions des acceptions de ces concepts.

\section{Des évolutions parallèles des concepts d'adaptation et capabilités}

Jusque dans les années 1990, l'adaptation n'occupait qu'une place mineure dans les rapports issus des sommets internationaux relatifs à l'environnement, ou de ceux du GIEC ${ }^{4}$. À partir de 1996, l'adaptation est vue comme un «ajustement» (Basset et Fogelman, 2013) : il s'agit d'identifier les risques naturels liés au changement climatique, définir le degré de risque que les individus sont prêts à accepter et déterminer les ajustements qu'ils accepteraient de faire en réponse aux effets du changement climatique. Puis, en 2001, l'adaptation selon le GIEC est «réformiste» car si elle est toujours considérée comme un ajustement, elle nécessite de réorienter les priorités socio-économiques et politiques. En 2014, nous estimons que le GIEC fait un pas supplémentaire vers une adaptation «transformationnelle » en considérant que « la planification et la mise en œuvre des mesures d'adaptation à tous les niveaux de gouvernance sont conditionnées par les valeurs et les objectifs de la société et par sa perception des risques » (Field et al., 2014). L'adaptation est toujours considérée sous la forme de stratégies et d'actions possibles, mais le GIEC estime désormais que les valeurs de la société doivent être prises en compte.

\footnotetext{
4 Elle était initialement considérée comme une stratégie «laxiste et fataliste» soutenue par les opposants du protocole de Kyoto (Marquet et Salles, 2014).
}

Le concept de capabilités a également connu une évolution dans ses acceptions depuis sa première occurrence. Initialement introduit par Sen (1999), le concept des capabilités critique et prolonge l'approche essentialiste de la justice développée par Rawls (2009): l'accès aux biens premiers, la levée du voile de l'ignorance et la délibération sur les règles d'équité ne suffisent pas à garantir la justice; les individus doivent également pouvoir être en capacité d'effectuer les mêmes actions. À ce titre, Sen (1999) définit la capabilité comme «la possibilité pour les individus de faire des choix parmi les biens qu'ils jugent estimables et de les atteindre effectivement ». Si cette approche permet de reconnaître des droits, Nussbaum (2000) défend l'idée qu'elle doit être accompagnée d'une philosophie éthique inscrite dans des pratiques démocratiques. L'approche par les capabilités n'est plus seulement une «approche comparative de l'évolution de la qualité de vie », mais aussi une «[théorisation] de la justice sociale élémentaire» (Nussbaum, 2000). Les capabilités selon Nussbaum représentent donc les possibilités saisies par des individus ou groupes de transformer leur relation avec l'environnement, en accord avec leurs principes éthiques, en fonction du contexte social, économique ou politique (Blanc et Laigle, 2018). La prise en compte des capabilités permet également de réfléchir à l'espace écologique, de manière que celles valorisées par les populations ne soient pas menacées par l'évolution de cet espace et des activités qui s'y exercent (Flipo, 2005).

\section{Faire le lien entre capabilités et adaptation}

Plusieurs études ont d'ores et déjà proposé d'effectuer un lien entre adaptation et capabilités. Si l'individu n'a pas les capabilités nécessaires pour faire face aux risques et «remonter la pente», il est vulnérable; en revanche, si grâce à ses capabilités il parvient à trouver des stratégies visant à se protéger contre le risque et à renforcer ses capabilités, on dit qu'il est «résilient» (Rousseau, 2007). Afin de déterminer les capabilités qui sont nécessaires pour faire face à des événements climatiques extrêmes, une méthodologie statistique est parfois adoptée, typologique, ou une méthodologie par les récits ${ }^{5}$. Dans le cadre de l'élaboration de stratégies d'adaptation transformationnelle toutefois, nous avons souligné la nécessité de ne pas seulement chercher à identifier les risques et les causes de la vulnérabilité, soit «s'adapter à quoi », mais aussi de réfléchir à «adapter quoi », soit à identifier les capabilités valorisées par la population.

\footnotetext{
${ }^{5}$ Les articles suivants adoptent par exemple respectivement les approches citées: Rousseau (2007); Lallau (2008); Benitez et Reghezza (2018).
} 
D'autres travaux ont, par exemple, proposé d'ajouter un principe de précaution sociale au principe de précaution environnementale, afin que les libertés individuelles ne soient pas menacées par les politiques de préservation environnementale (Ballet et al., 2011). Si elle est plus réformiste, en ce qu'elle permet d'inscrire de nouvelles priorités sociales et qu'elle envisage de nouveaux droits, une telle approche ne propose pas encore de réflexion quant aux capabilités collectives. Or, pour passer d'une adaptation incrémentale à une adaptation transformationnelle, impliquant des changements au niveau des individus, des institutions et des cultures, la mobilisation des capabilités individuelles et collectives est nécessaire (Pelling et al., 2014).

Afin d'accompagner une adaptation transformationnelle, les capabilités doivent également être en mesure de faire le lien avec les valeurs, les principes éthiques et moraux des populations, leurs possibilités de les exprimer démocratiquement et de les traduire en action. D'une importance cruciale, parce qu'une capabilité ne compte "que si une personne la valorise», ce lien encourage la participation et l'engagement des parties concernées (Alkire et Deneulin, 2009).

Comment appréhender cette approche transformatrice? Le récit devient pour nous un outil essentiel, puisque c'est aussi à travers lui que les valeurs et principes s'expriment. Sachant toutefois que tout récit emprunte à un moment de préfiguration des histoires par les acteurs eux-mêmes, qui donnent sens à leurs actes (Ricoeur, 1983 cité par Cefaï, 2010, p. 548), il est important de comprendre qui peut participer au récit, comment et de quelle manière ces personnes ont été familiarisées à l'adaptation (Chezel, 2018). Nous montrerons dans cet article qu'en dépit de la présence d'outils tels que la participation publique et l'empowerment, une telle mise en récit au sein des stratégies locales d'adaptation est actuellement très limitée.

\section{Participation publique et empowerment, pour favoriser l'émergence de récits}

La prise en compte des capabilités dans les politiques locales repose sur deux entrées principales : la première, fortement associée au développement durable, est la participation publique. Relevant la plupart du temps d'une initiative politique, et rarement directement citoyenne, elle consiste notamment à informer et à entendre les populations locales dans divers débats et arènes.

Si l'action collective est dominée par l'injonction participative émanant des pouvoirs publics (Bacqué et Gauthier, 2011) aux dépens de la reconnaissance des mouvements spontanés et de l'action directe plus ou moins formalisée, les politiques publiques ont su faire place à l'idée d'empowerment (Bacqué et Biewener, 2014), entérinant l'acquisition d'une légitimité démocratique et d'une capacitation des associations face à la puissance publique, souvent gouvernée par les savoirs de «l'expert» professionnel (Pestre, 2011). Cette reconnaissance passe notamment par des appels à projets citoyens. On peut, cependant, s'interroger sur la place réelle qu'accordent aux citoyens ainsi qu'à la possibilité de développer leurs capacités ces appels à projets variés dans leurs contenus. Sont-ils à même de faire le lien entre politiques publiques et mobilisations citoyennes, cellesci étant porteuses de propositions ou de projets politiques extrêmement variés (manifestation, occupation d'espaces, camps spontanés, guerrilla gardening, jardins sauvages, etc.)?

Dans un cas ou dans l'autre, la question est celle de la mise en relation de possibilités et de capacités identifiées localement, que cette mise en relation résulte de ces mobilisations bottom-up ou d'une incitation politique top-down. Dans les deux cas, notre objectif consiste à identifier si la participation publique ou les appels à projets permettent l'émergence de nouvelles possibilités individuelles, mais aussi de valeurs collectives, et si les plans élaborés grâce à la participation publique ou les projets correspondent à une adaptation ou d'ajustement ou transformationnelle.

Les possibilités individuelles peuvent être développées dans les plans et stratégies élaborés grâce à la participation publique ou les appels à projets en identifiant de nouvelles opportunités et possibilités d'action pour les citoyens.

Afin de permettre l'émergence de valeurs et principes collectifs, étape essentielle pour faire le lien avec une adaptation transformationnelle, les dispositifs participatifs devraient répondre à au moins trois contraintes: l'information mise à disposition, pour établir un choix raisonné; être partie prenante pour exprimer les raisons de valoriser tel ou tel objectif; la coproduction de savoirs pour avoir un pouvoir effectif dans la définition et l'atteinte des objectifs (Ballet et al., 2015). L'échelle d'Arnstein (1969) nous permettra de déterminer si les dispositifs participatifs étudiés répondent à de telles contraintes. Il s'agira d'analyser le lien entre le dispositif participatif mis en œuvre, les capabilités qui en découlent et le type d'adaptation proposé par les plans étudiés.

\section{Terrains, outils et méthode}

Les cas d'étude ont été sélectionnés suite aux résultats du projet CIVIC'ACT financé par le programme de recherche Politiques de la Terre. Ce projet a abouti à une classification des 131 communes de la métropole du Grand Paris en fonction de variables 
Tab. 1. Les outils institutionnels porteurs d'une stratégie d'adaptation locale au sein des trois territoires étudiés.

\begin{tabular}{|l|l|l|l|}
\hline & $\begin{array}{l}\text { Aubervilliers / Plaine } \\
\text { Commune }\end{array}$ & $\begin{array}{l}\text { Gonesse / Roissy Pays } \\
\text { de France }\end{array}$ & Paris \\
\hline Plans climat & $\begin{array}{l}\text { Plan climat de Plaine } \\
\text { Commune (2010) }\end{array}$ & $\begin{array}{l}\text { Plan climat de Roissy Pays de } \\
\text { France (en cours d'élaboration) }\end{array}$ & $\begin{array}{l}\text { Plans climat (2007, 2012 et } \\
\text { 2018) }\end{array}$ \\
\hline $\begin{array}{l}\text { Plans } \\
\text { «transversaux» }\end{array}$ & $\begin{array}{l}\text { Agenda 21 de Plaine } \\
\text { Commune (2012) } \\
\text { Agendas 21 d'Aubervilliers } \\
\text { (2012 et 2018) }\end{array}$ & $\begin{array}{l}\text { Agenda 21 de Gonesse (2012) } \\
\text { Agenda 21 de Roissy Pays de } \\
\text { France (en cours d'élaboration) }\end{array}$ & $\begin{array}{l}\text { Stratégie d'adaptation (2015) } \\
\text { Stratégie de résilience (2017) }\end{array}$ \\
\hline Appels à projets & $\begin{array}{l}\text { Terres d'Avenir (Plaine } \\
\text { Commune) }\end{array}$ & $\begin{array}{l}\text { Inventons la Métropole du } \\
\text { Grand Paris }\end{array}$ & Parisculteurs \\
\hline
\end{tabular}

socio-environnementales ${ }^{6}$ : l'occupation du sol (incluant l'exposition aux polluants); une analyse socio-économique et démographique; les résultats électoraux; le nombre d'associations environnementales (Antolinos-Basso et al., 2020). Antolinos-Basso et ses collègues montrent que «les groupes [ndlr: les associations et collectifs à visée environnementale] apportent des réponses contextualisées aux insuffisances perçues de leur cadre de vie », et donc qu'à ce titre le type d'engagement environnemental est dépendant du profil des différentes communes.

Nous avons choisi d'étudier des territoires appartenant aux trois catégories de communes identifiées, pour comprendre si les spécificités territoriales mènent ou non à l'élaboration de stratégies d'adaptation différenciées, intégrant différemment les capabilités. Trois territoires ont ainsi été sélectionnés :

- la ville d'Aubervilliers, dans l'établissement public territorial Plaine Commune, appartient à la première classe de communes, correspondant à des communes péricentrales défavorisées, présentant un fort taux d'imperméabilisation des sols, un fort taux de défaveur sociale ainsi qu'un nombre d'associations variable ;

- le $17^{\mathrm{e}}$ arrondissement parisien appartient à la seconde classe, marquée par un taux élevé d'imperméabilisation des sols, une population socialement favorisée et un grand nombre d'associations environnementales ;

\footnotetext{
$\overline{6}$ Le projet CIVIC'ACT, tout en soulignant ses limites, a souhaité valoriser l'interopérabilité des données quantitatives (issues des différentes bases de données) et qualitatives (issues de 52 entretiens avec des associations et collectifs locaux à visée environnementale, ainsi que d'observations locales au sein de 31 des 131 communes étudiées).
}

- la ville de Gonesse, au sein de la communauté d'agglomération Roissy Pays de France, appartient à la troisième classe, se distinguant par une proportion importante de sols perméables, des communes généralement aisées ainsi qu'un faible nombre d'associations environnementales.

Afin d'analyser l'évolution des stratégies d'adaptation dans ces territoires, nous avons étudié principalement trois types d'outils institutionnels: les plans climat, les plans que nous qualifions de «transversaux », et les appels à projets à deux échelles, communale et communauté de communes. Le tableau 1 regroupe les outils étudiés en fonction des territoires sélectionnés.

Les plans climat énergie territoriaux (PCET) sont obligatoires depuis 2012 suite à la loi Grenelle II pour les établissements publics de coopération intercommunale (EPCI) de plus de 50000 habitants. Depuis 2017, une dimension "air» a été ajoutée, rendant les plans climat-air-énergie territoriaux obligatoires pour les EPCI de plus de 50000 habitants et, au 31 décembre 2018, pour les EPCI de plus de 20000 habitants. Les villes d'Aubervilliers et de Gonesse ne sont pas concernées par ces obligations légales, contrairement aux communautés de communes Plaine Commune et Roissy Pays de France. L'adaptation peut également être abordée dans le cadre de plans «transversaux » qui font le lien entre les problématiques climat et d'autres thématiques, tels que les Agendas 21 qui développent un programme d'actions en faveur du développement durable, réalisés de manière volontaire par les communautés ou les communes qui les choisissent. Nous nous sommes également intéressés à trois appels à projets : Terres d'Avenir, Inventons la Métropole du Grand Paris et Parisculteurs. 
Pour analyser la place des capabilités au sein des stratégies d'adaptation locales, nous nous sommes appuyés sur une analyse détaillée des plans étudiés, des entretiens avec des agents administratifs et des élus des territoires concernés ainsi que des visites de terrain. Nous avons également mené une vingtaine d'entretiens avec des responsables associatifs en 2018, et organisé deux ateliers CAPADAPT à Aubervilliers (le 23 février 2019) et Paris (le 27 février 2019) qui nous ont notamment permis de recueillir le ressenti des habitants quant aux plans et appels à projets locaux.

Les stratégies d'adaptation locales sont souvent distribuées dans des plans séparés. Le plan climat, parce qu'il est obligatoire et parce qu'il est recommandé qu'il tienne compte d'un bilan des gaz à effet de serre, semble moins favorable à l'émergence des récits citoyens et des valeurs collectives que l'Agenda 21, qui a un caractère transversal et est à l'initiative des territoires. Les appels à projets apparaissent comme une mise en œuvre concrète des stratégies adoptées localement, susceptible de renforcer les capabilités citoyennes. Nous analyserons successivement le type d'adaptation proposée et la place accordée aux capabilités au sein de chacun de ces outils.

\section{Les plans climat: une faible prise en compte des capabilités pour des stratégies d'adaptation de type ajustement}

\section{Les plans climat parisiens}

Au travers des différents plans climat adoptés par la ville de Paris, en 2007, 2012, puis 2018 l'adaptation fait l'objet d'une stratégie à part, disposant d'une section séparée dans le plan. En 2007, la stratégie d'adaptation est pensée comme complémentaire de la stratégie d'atténuation, mais demeure une priorité secondaire: la stratégie d'atténuation constitue «le corps central du plan climat ${ }^{7}$. La stratégie d'adaptation identifie d'abord les vulnérabilités de la ville en s'appuyant sur le Livre blanc: l'exposition de la population à la canicule, ainsi que le risque de crue de la Seine. Le plan canicule est alors l'action prioritaire de cette première stratégie d'adaptation. S'y ajoutent un autre plan de prévention des risques, le plan de prévention des risques d'inondation; des mesures sectorielles, comme le souhait de développer une filière professionnelle pour améliorer le confort d'été; et des actions visant tant l'atténuation que l'adaptation, comme la compensation carbone ainsi

\footnotetext{
${ }^{7}$ Mairie de Paris, 2007. Plan climat de Paris. Sauf erreur de notre part, ce plan climat n'est au moment de la rédaction de notre article plus disponible sur le site de la mairie de Paris.
}

qu'un «important programme de végétalisation de la capitale». Cette première stratégie est donc à classer dans la catégorie des approches utilisées par les climatologues et les écologues, en ce qu'elle cherche principalement à limiter les risques associés au changement climatique.

La partie adaptation du plan climat de $2012^{8}$ continue de s'intéresser majoritairement aux risques: canicules, effets d'îlots de chaleur urbains; inondations, sécheresses, potabilité.

Le plan climat parisien a été révisé en $2018^{9}$, suite à l'élaboration de deux stratégies (d'adaptation et de résilience), et pour se conformer aux nouvelles obligations légales. La partie adaptation a peu évolué sur le fond. Sur la forme, le nouveau plan climat entérine la vision de l'adaptation comme résilience: le mot d'adaptation a disparu et la partie s'intitule «Une ville résiliente qui veille à la qualité de son cadre de vie». Le préambule de cette partie fait d'ailleurs une large place au renvoi vers la stratégie de résilience parisienne. Les risques, bien que dissimulés au sein d'un plan qui s'organise autour des quatre éléments, air, feu, terre, eau, sont toujours présents : par exemple l'air renvoie aux pics de pollution, le feu aux canicules et incendies.

Les solutions pour l'adaptation sont donc toujours largement pensées de manière sectorielle: une réponse pour chaque risque, en faisant appel aux professionnels du secteur concerné pour trouver une réponse adaptée.

Pour élaborer le premier plan climat de 2007, le diagnostic n'a pas été réalisé avec les habitants : l'étude technique a précédé le dispositif participatif. Dès 2004, avant le lancement du plan, la ville de Paris a en effet testé l'outil Bilan Carbone (Ademe) pour évaluer les émissions de différents acteurs au sein de la ville. La «concertation» ne porte pas sur l'objectif principal, le « facteur $4 »$, mais concerne principalement les actions à mettre en place. Les citoyens ont une place limitée dans ce processus. Les ateliers ont majoritairement réuni des acteurs clés et une centaine de citoyens volontaires. La ville de Paris a cherché l'exemplarité dans ce premier plan d'actions, en montrant d'abord que l'administration pouvait atteindre les objectifs définis. Les thèmes étaient par ailleurs prédéfinis, largement calqués sur les principaux pôles émetteurs identifiés dans le Bilan Carbone. Ce choix d'une approche sectorielle, s'appuyant sur les acteurs clés de différents secteurs concernés pour trouver des solutions ne semble pas non plus avoir été mis à la discussion. En termes de

\footnotetext{
${ }^{8}$ Mairie de Paris, 2012. Plan climat énergie de Paris, https:// api-site-cdn.paris.fr/images/70921.

${ }^{9}$ Mairie de Paris, 2018. Plan climat de Paris, https://api-sitecdn.paris.fr/images/102678.
} 
contenu, une partie «communication et sensibilisation» concerne directement les Parisiens. Il s'agit de les informer et de les sensibiliser à la cause. A contrario, il ne s'agit pas de s'appuyer sur des initiatives citoyennes ou de les renforcer. Cette démarche participative a donc un aspect thérapeutique au sens d'Arnstein (1969): il s'agit de convaincre les participants de la légitimité de la cause ${ }^{10}$.

La révision du plan climat adopté en 2012 est très largement issue d'une étude technique traitant de la robustesse et de la vulnérabilité de Paris face au changement climatique et à la raréfaction des ressources. La place accordée à la participation citoyenne y est marginale.

Le nouveau plan climat-air-énergie de 2018 n'est pas considéré comme une simple actualisation, mais comme un nouveau plan, avec de nouveaux objectifs, notamment celui de neutralité carbone pour 2050. Les tendances précédentes se confirment : l'objectif de neutralité n'est pas discuté avec les citoyens. Les professionnels sont considérés comme ayant un rôle majeur à jouer pour atteindre cet objectif. La faible progression du nombre de commentaires reçus sur le site internet depuis le premier plan confirme que les discussions autour des plans climat parisiens sont l'apanage d'un groupe d'initiés. Toutefois, l'ajout de la conférence de citoyens et d'acteurs du monde associatif souligne une nouveauté majeure: la prise de conscience, confirmée par les agents administratifs, que l'objectif ambitieux défini par la ville ne pourra être atteint sans les citoyens. La conférence de citoyens $^{11}$ a, par exemple, permis de souligner la méconnaissance qu'ont les citoyens tant des objectifs que des actions mises en place par la ville de Paris, et d'aborder des pistes pour mieux les inclure.

Avec l'objectif neutralité carbone, la ville a donc pris la mesure de la nécessité de s'appuyer sur les citoyens :

«On était déjà plutôt bons, je pense qu'on est devenu même très bons, sur vraiment la planification de documents stratégiques, les différentes actions qui sont nécessaires pour atteindre nos objectifs. Et je pense que maintenant, notre plus gros challenge, que ce soit dans l'adaptation ou dans une autre, c'est qu'il y ait une appropriation réelle, des différentes parties prenantes, et aussi des citoyens bien sûr, sur ces questions-là pour qu'on puisse atteindre nos objectifs $»^{12}$.

\footnotetext{
${ }^{10}$ Même si la démarche de son ensemble ne peut pas se limiterà de la thérapie, puisque les citoyens sont entendus (consultation et concertation).

11 Mairie de Paris, 2016. Conférence de citoyens Paris championne du Climat: quelles contributions individuelles et collectives pour changer nos modes de vie?, https://parcs. hypotheses.org/files/2016/11/charte-paris-climat-ifop-8-octo bre-2016.pdf.

12 Entretien avec deux membres de la division ClimatEnergies et économie circulaire de la mairie de Paris, à Paris le 18 décembre 2017.
}

Les plans climat adoptés dans un premier temps se focalisent donc peu sur les capabilités des Parisiens. L'absence de discussion sur les objectifs et priorités relatifs à l'adaptation lors des processus participatifs ne permet pas de faire émerger des valeurs collectives. Les citoyens n'y sont pas considérés comme des acteurs majeurs de la transition: les professionnels et l'administration sont les récipients majeurs des plans climat. Nous verrons toutefois que la ville tente de s'adresser aux citoyens autrement, via notamment des appels à projets et comités de volontaires.

\section{Le plan climat de Plaine Commune}

Dès 2010, soit avant l'obligation légale comme pour Paris, Plaine Commune s'est dotée d'un premier plan climat $^{13}$. Il relève principalement d'une approche ingénieriste et technique émanant de la collectivité locale. Centré essentiellement sur l'atténuation, le plan a «un objectif prioritaire», «la réduction des gaz à effet de serre » pour répondre aux objectifs du paquet climaténergie européen ${ }^{14}$. L'approche retenue est sectorielle: le plan d'action décline les objectifs par secteur (résidentiel, déplacement de personnes, transport de marchandises, secteur tertiaire, industrie) tout en affirmant une vision et des valeurs liées à la solidarité avec les plus démunis. Celle-ci consiste à «mettre à disposition la transition énergétique pour les plus précarisés », mais aussi à améliorer le confort d'été dans les quartiers d'habitat social, à recréer des corridors écologiques entre les quartiers denses et avec le nord du territoire, par exemple en favorisant des liaisons douces et la marche.

Comme la ville de Paris, le facteur 4 est adopté en fonction d'objectifs nationaux. Il n'est pas non plus discuté avec les citoyens. Comme à Paris également, la démarche d'élaboration du plan a démarré avec des études techniques: le bilan énergétique et le bilan carbone propres au territoire de Plaine Commune. Le plan climat lui-même a été élaboré avec le conseil de développement et un comité citoyen, composé de 60 volontaires, qui se sont réunis à cinq reprises. Si ces

13 Plaine Commune, 2010. Plan climat énergie de Plaine Commune, https://plainecommune.fr/fileadmin/user_upload/ Portail_Plaine_Commune/LA_DOC/THEMATIQUES/Ecolo gie_urbaine/Plan-Climat-Energie-2010-2020.pdf.

${ }_{14}$ Une réduction des émissions de gaz à effet de serre de $20 \%$ par rapport à 1990; une contribution des énergies renouvelables de $20 \%$ à la consommation d'énergies finales en 2020 (23\% pour la France); une économie d'énergie de $20 \%$ sur la consommation primaire par rapport au scénario tendanciel. 
volontaires saluent la méthode, ils ont souligné plusieurs limites $^{15}$ :

- les sessions sont parfois trop denses, il y a trop d'informations et pas assez de discussions ;

- l'outil collaboratif (page Wikipédia) n'est pas adapté ;

- il n'y a pas de points d'avancement réguliers avec les autres groupes de travail de l'agglomération;

- il n'y a pas assez de débats et de controverses et peu d'échanges sur les actions proposées;

- il en découle une contribution collective incomplète : peu d'actions sont portées par les citoyens et les associations.

L'initiative du comité citoyen pâtit donc d'un manque de discussions et de débats qui n'a pas favorisé l'émergence de valeurs collectives. Plutôt que sur des principes et valeurs, le plan s'appuie sur une approche sectorielle (une réduction d'émissions par secteur) qui ne permet pas aux citoyens de proposer des actions qu'ils pourraient mettre en place, ni de réfléchir collectivement aux manières dont ils pourraient devenir acteurs de l'adaptation.

\section{Roissy Pays de France: un plan climat à définir}

La communauté d'agglomération de Roissy Pays de France a été constituée au $1^{\mathrm{er}}$ janvier 2019 par une décision préfectorale qui a fusionné deux communautés déjà existantes. Depuis le début de l'année 2018, elle s'est simultanément engagée dans l'élaboration d'un schéma de cohérence territoriale (SCOT), d'un Agenda 21 et d'un plan climat. Si ce calendrier paraît propice à l'élaboration d'une définition de l'adaptation propre à la communauté d'agglomération, plusieurs obstacles laissent néanmoins penser qu'il sera difficile de parvenir à une définition transversale et transformationnelle de cette adaptation. Le SCOT et le plan climat ne seront pas élaborés par les mêmes prestataires, parce que deux directions administratives différentes, ayant chacune développé leurs critères à respecter, supervisent leur élaboration ${ }^{16}$. La direction du développement durable préfère travailler par projets plutôt que par plans, parce qu'elle considère que les projets sont plus aptes à capter l'attention politique. Une telle approche semble peu susceptible de favoriser l'émergence de valeurs et principes collectifs.

\footnotetext{
15 Plaine Commune, 2010. Avis du Comité Citoyen pour l'élaboration du Plan Climat Energie de Plaine Commune, https://plainecommune.fr/fileadmin/user_upload/Portail_Plai ne Commune/LA DOC/THEMATIQUES/Ecologie urbaine/ pce_comite_final.pdf.

${ }^{16}$ Entretien avec un membre de la direction du développement durable de Roissy Pays de France, à Sarcelles le 3 avril 2018.
}

\section{Les plans «transversaux »: une implication citoyenne pour une adaptation réformiste, sans émergence de valeurs collectives}

\section{Les stratégies d'adaptation et de résilience à Paris}

Deux stratégies sont pensées comme venant compléter les plans climat parisiens en s'intéressant principalement à l'adaptation : la stratégie d'adaptation de $2015^{17}$ et la stratégie de résilience de $2017^{18}$.

La stratégie d'adaptation vient plus compléter que révolutionner la stratégie initiée dans les plans climat. Alors que ce nouveau document devait favoriser l'élaboration d'une stratégie intégrée avec une liste d'actions priorisée, les citoyens y ont joué un rôle mineur. Les agents administratifs confirment le rôle majeur de l'étude sur la vulnérabilité et robustesse de Paris dans la rédaction de cette stratégie ${ }^{19}$. La stratégie d'adaptation de 2015 est en effet largement issue d'une nouvelle étude technique: l'étude-diagnostic des forces et faiblesses de Paris face au changement climatique et à la raréfaction des ressources. En conséquence, les priorités s'étoffent, mais ne changent pas: la priorité principale reste de protéger les Parisiens face aux événements climatiques extrêmes. Ensuite s'ajoute l'idée de préserver les ressources (en eau en premier lieu) et de renforcer la solidarité.

A contrario, la stratégie de résilience a été élaborée avec 800 parties prenantes, issues de différents secteurs associatifs et professionnels, qui ont identifié six enjeux majeurs: le dérèglement climatique est en troisième position, après les inégalités socio-économiques et le risque terroriste, avant la pollution de l'air, les risques liés au fleuve et la gouvernance territoriale. Le diagnostic et la présentation des enjeux ne reposent toutefois pas sur des récits citoyens. La présentation de l'enjeu climatique démarre ainsi avec une citation d'un célèbre climatologue, Jean Jouzel, puis avec des chiffres (comme l'augmentation de la température moyenne de 2 à 4 degrés) et des «effets tangibles». La stratégie de résilience vient ensuite confirmer la vision de l'adaptation comme gestion des risques. Le permis de végétaliser, déjà en place, est évoqué, mais pas ses bénéfices au

\footnotetext{
17 Mairie de Paris, 2015. Stratégie d'adaptation, https://apisite.paris.fr/images/76270.

18 Mairie de Paris, 2017. Stratégie de résilience de Paris, https://api-site-cdn.paris.fr/images/95335.

19 Entretien avec deux membres de la division ClimatEnergies et économie circulaire de la mairie de Paris, à Paris le 18 décembre 2017.
} 
regard de l'adaptation. La «[mobilisation] de l'intelligence et des ressources collectives », dans le troisième pilier relatif à la ville en transition, propose de créer un centre des ressources, un observatoire pour connaître les impacts, de mobiliser les acteurs du numérique et de créer de nouveaux mécanismes de financement. Les actions envisagées reposent donc peu sur les citoyens.

\section{L’Agenda 21 de Plaine Commune}

À Plaine Commune, si les stratégies d'adaptation de type ajustement ou gestion des risques sont privilégiées dans le cadre des plans climat, l'Agenda 21 semble ouvrir la voie à une adaptation plus réformiste.

L'Agenda 21 de Plaine Commune ${ }^{20}$ entérine l'idée d'une adaptation qui prend en compte les inégalités sociales et territoriales, en proposant notamment le développement d'un pôle de compétences pour lutter contre la précarité énergétique.

Les citoyens ont été consultés largement pour élaborer l'Agenda: l'objectif de «toucher 1\% des habitants et usagers du territoire de Plaine Commune», bien plus ambitieux que ce qui a été fait pour les plans climat, a été atteint. La démarche n'a pas touché que des initiés: des efforts ont été faits pour aller vers les résidents et les usagers, via le biais de questionnaires, et des efforts ont également été faits pour entendre les populations «en difficultés». L'Agenda 21 fait ainsi place à la parole citoyenne, tant pour établir le diagnostic que pour définir le plan d'actions. Les citoyens demandent, par exemple, une meilleure éducation à l'environnement. Un inventaire des pratiques est établi, suivi d'une déclaration d'intentions relative au changement de pratique: «les habitants interrogés se disent prêts à faire des efforts pour modifier leurs pratiques quotidiennes: moins consommer d'eau $(93 \%)$ et récupérer l'eau de pluie $(75 \%)$, chauffer moins (42\%), utiliser les modes doux, donner de leur temps ». Que faudrait-il pour passer de l'intention à l'action? Comment faire grandir les capabilités citoyennes? Ces questions ne sont pas évoquées, ni les raisons pour lesquelles les citoyens considèrent qu'il est important de se préoccuper des questions climatiques à Plaine Commune. En l'absence d'une telle analyse, les citoyens ont eu des difficultés à proposer des actions concrètes : ils sont peu mobilisés pour mettre en œuvre la partie adaptation, alors que dans le reste de l'agenda, de nombreuses propositions d'action s'appuient sur leurs initiatives.

\footnotetext{
${ }^{20}$ L'Agenda 21 de Plaine Commune est présenté sous la forme de quatre livrets, disponibles sur le site de Plaine Commune : https://plainecommune.fr/institution/le-projet-de-territoire/ agenda-21/.
}

\section{Les Agendas 21 de Gonesse et Aubervilliers}

À l'occasion de la COP 21 en 2015, l'ensemble des villes de Plaine Commune ont signé une «résolution d'engagements communs pour la sauvegarde du climat et l'amélioration de la qualité de vie ${ }^{21}$. Elle met au premier plan la coopération pour «inventer le territoire de demain, sobre en carbone et apaisé » et « construire un autre modèle urbain adapté aux changements climatiques, sensible à la biodiversité [...] en inventant de nouvelles formes de coopération entre collectivitéssociété civile-entreprises ». Cette résolution engage les signataires à « décliner ces orientations en un programme d'actions concrètes ». La charge de la définition de la stratégie d'adaptation est alors largement reportée sur les communes.

Différents élus d'Aubervilliers se sont montrés sensibles au lien entre précarité et environnement, en évoquant notamment le besoin d'espaces verts pour les personnes vivant dans des logements petits, sociaux, ou insalubres $^{22}$. Ils déplorent cependant un manque de moyens pour mettre en œuvre des solutions. Le développement durable n'apparaît pas comme une priorité pour les élus de Gonesse, qui insistent sur le développement de grands projets d'aménagement dans l'optique de lutter contre le chômage ${ }^{23}$. Ces différentes sensibilités semblent bien avoir un impact sur la définition des stratégies d'adaptation: la comparaison des Agendas 21 des communes d'Aubervilliers et Gonesse montre pour le premier une meilleure inclusion des citoyens pour une stratégie d'adaptation réformiste.

D'après l'Agenda 21 de Gonesse ${ }^{24}$, l'élaboration du diagnostic a été partagée avec les Gonessiens, via la distribution de questionnaires lors d'un forum citoyen. Puis des ateliers ont été organisés. L'axe 1 (Favoriser les changements de comportements individuels et collectifs)

\footnotetext{
21 Plaine Commune, 2015. Résolution d'engagements communs pour la sauvegarde du climat et l'amélioration de la qualité de vie à Plaine Commune, https://plainecommune.fr/ fileadmin/user_upload/Portail_Plaine_Commune/LA_DOC/ THEMATIQUES/Ecologie urbaine/resolution climat.pdf.

22 Sept élus d'Aubervilliers ont été interrogés dans le cadre du projet CAPADAPT du 4 au 21 septembre 2018. Tous ces élus se sont montrés sensibles au lien entre environnement et précarité

23 Les élus de Gonesse n'ont pas souhaité nous rencontrer. Nous nous sommes donc notamment appuyés sur le document suivant: Jean-Pierre Blazy, 2019. Discours de Jean-Pierre Blazy lors des voeux à la population. http:/www.villegonesse.fr/news/discours-de-jean-pierre-blazy-lors-desvoeux-\%C3\%A0-la-population.

24 Le document nous a été transmis par des agents administratifs : Ville de Gonesse, 2012. Gonesse 21: L'Agenda 21 Local. Adopté lors de la séance du Conseil Municipal du 29 Mars 2012 -délibération $\mathrm{N}^{\circ} 49$.
} 
concerne les citoyens. Toutefois, comme le reste du plan, les actions de cette partie sont scindées entre celles qui ont un objectif socio-économique et celles qui ont un objectif environnemental. Très peu correspondent au second objectif.

À Aubervilliers ${ }^{25}$, a contrario, la durabilité est intégrée, vue comme devant être transversale, et l'articulation est déjà prévue et engagée avec Plaine Commune. Un bilan de l'Agenda 21 d'Aubervilliers effectué en 2015 souligne toutefois que le développement ou la coordination d'actions citoyennes aidant à l'adaptation fait partie des objectifs non atteints, par exemple: [Des actions] visant à améliorer la nature en ville sont engagées mais sont encore à développer avec la mise en relation des 21 jardins partagés ou des apiculteurs des 5 ruchers locaux. L'Agenda 21 d'Aubervilliers a été révisé en $2018^{26}$, en s'appuyant à la fois sur des ateliers citoyens et des ateliers institutionnels. Au sein des ateliers citoyens, 30 associations ont formulé 86 propositions. Le dialogue institutionnel, entre l'agence locale de l'énergie, Plaine Commune, des techniciens et des élus, a conduit à émettre 174 propositions. Les élus ont fait le choix de retenir un nombre réduit de 20 actions : 12 issues des ateliers citoyens et 8 des ateliers institutionnels.

La focalisation sur un programme d'actions concrètes délaisse un diagnostic partagé, le choix de valeurs et principes communs. Tenter de comprendre ce dont les citoyens auraient besoin pour se mobiliser ainsi que les capabilités qu'ils valorisent sont des étapes négligées dans l'ensemble des plans étudiés. Elles semblent pourtant essentielles au vu des difficultés à proposer ou mettre en œuvre des actions citoyennes.

Par ailleurs, les élus conservent le pouvoir décisionnaire. La participation citoyenne, dans le cadre de l'Agenda 21 d'Aubervilliers, relève ainsi d'après l'échelle d'Arnstein de l'implication, mais ne permet pas encore de donner un véritable "pouvoir aux citoyens », qui consisterait à leur permettre de choisir avec les élus les actions à mener. Si des actions issues de propositions citoyennes sont incluses dans le plan, la non-inclusion des citoyens dans le choix des actions freine les réflexions quant à l'élaboration d'une stratégie cohérente pour le développement des capabilités citoyennes. Elle engendre également une lassitude et un découragement des citoyens : les responsables associatifs rencontrés à Aubervilliers ${ }^{27}$ nous ont par exemple fait

\footnotetext{
${ }^{25}$ Le site Agenda 21 de la mairie d'Aubervilliers: http:// agenda21.aubervilliers.fr/.

${ }^{26}$ Le processus de révision ainsi que les grandes orientations du nouvel agenda 21 d'Aubervilliers ont été présentés par le chargé de missions Agenda 21 et PCAET, lors d'un entretien à Aubervilliers le 20 juin 2018.

27 Dix responsables associatifs ont été interrogés lors d'entretiens semi-directifs à Aubervilliers entre mai et septembre 2018.
}

savoir qu'ils considèrent que les actions retenues dans l'Agenda 21 leur semblent largement insuffisantes (comme l'action de planter 500 arbres en 10 ans).

Les limites des processus participatifs sont connues. Les dispositifs participatifs feraient de manière générale «beaucoup de bruit pour rien » car les effets escomptés se produisent rarement (Blondiaux et Fourniau, 2011). Les processus participatifs étudiés ne dérogent pas à de telles observations. Notre étude souligne que ces limites engendrent plusieurs problèmes pour la prise en compte des capabilités dans les stratégies d'adaptation locales. Les citoyens ne participent pas à la définition d'objectifs, ni à l'adoption de valeurs et de principes communs. Il n'y a pas d'analyse relative à ce qui favoriserait leur engagement, leur permettrait d'agir pour un futur qu'ils désirent. Si les récits ne sont pas encouragés lors de l'élaboration de stratégies d'adaptation locales, les appels à projets permettent-ils, par la pratique, un grandissement des capabilités grâce à l'échange d'expériences? Favorisent-ils l'émergence de valeurs communes?

\section{Les appels à projets et comités de volontaires, pour renforcer les capabilités citoyennes?}

L'appel à projet Terre d'Avenir a été mis en place suite à l'Agenda 21 de Plaine Commune. Les projets doivent concerner le territoire et ses habitants, porter si possible sur plusieurs quartiers ou plusieurs villes, être coopératifs et valoriser les ressources humaines locales et la diversité. Ils doivent être les plus économes possibles en ressources et en énergie et surtout répondre à au moins un axe de l'Agenda 21. À Plaine Commune, cet appel à projets a par exemple permis de :

«soutenir des associations qui vont dans le même sens que l'Agenda 21 et le plan climat et donner envie à d'autres associations de le faire. Exemple: on soutient l'association Bicyclo qui fait de l'insertion et de la réparation vélo, formation à la conduite de vélo, etc. On espère que cela fait des petits! Dans le plan d'actions du plan climat, il est dit qu'on va soutenir les acteurs de la transition. Il y a plusieurs trajectoires possibles pour soutenir ces acteurs. Exemple: Bellastock est arrivé pour faire leur festival sur l'Île-Saint-Denis, montrer la diversité de l'utilisation des matériaux. Comme on déconstruit et on urbanise, le maire a demandé si c'est possible de créer un laboratoire in situ $[\ldots] »^{28}$.

28 Entretien avec un membre de la délégation générale de l'écologie urbaine de Plaine Commune, à Saint-Denis le 30 janvier 2018. 
Les citoyens ont toutefois souligné une limite majeure de cet appel à projets ${ }^{29}$ : au lieu de favoriser l'échange d'expériences, les projets sont mis en compétition pour obtenir des fonds, ce qui n'encourage ni le développement des capabilités entre citoyens, ni l'émergence de valeurs collectives.

Le manque de moyens déploré par les élus albertivillariens conduit ainsi à une mise en compétition de projets citoyens, plutôt qu'à l'émergence d'une stratégie collective s'appuyant sur les citoyens.

À Gonesse, l'appel à projet «Inventons la métropole » incluait un terrain dans la commune, bien qu'il soit en dehors des limites du Grand Paris. Parmi les réponses, celle appelée CARMA (Coopération pour une ambition rurale et métropolitaine d'avenir) émanait d'un collectif d'associations et de professionnels opposé à l'urbanisation des terres agricoles du triangle de Gonesse incluse dans une zone d'aménagement concerté (ZAC) portée par la ville et un aménageur public. Ce projet proposait une alternative à l'urbanisation qui répond aux défis du changement climatique. Une partie du projet, sur 15 hectares du site, consistait à faire une expérience d'agroforesterie susceptible de fournir les cantines locales et de récupérer les déchets organiques. En protégeant la terre agricole, ce projet annonçait aussi réduire les risques de pic de chaleur et protéger la biodiversité tout en offrant des perspectives de développement économique et d'amélioration de qualité de la vie aux habitant ${ }^{30}$. Arrivé en phase finale du concours, le projet CARMA a pourtant été éliminé par un veto du maire de Gonesse qui lui a préféré un projet porté par un promoteur belge de bureaux avec toitures végétalisées. Ce cas montre à Gonesse un manque de dialogue entre élus et citoyens quant à l'établissement des valeurs et principes collectifs ainsi qu'une volonté des institutions de contenir les actions citoyennes liées au climat dans des domaines mineurs tels qu'ils sont décrits dans l'Agenda 21 de la commune.

À Paris, nombre d'appels à projets sont favorables à l'adaptation citoyenne, comme Parisculteurs, Réinventer Paris, ou encore Inventons la Métropole du Grand Paris qui souhaite par exemple valoriser la production et la consommation locales. Les recherches tendent toutefois à souligner le caractère anecdotique de ces appels à projets : le nombre et la taille de parcelles dans le cadre $\mathrm{du}$ projet Parisculteurs seraient «modestes, voire anecdotiques », et à ce titre ne permettent pas de constituer une alternative aux dynamiques d'approvi-

\footnotetext{
${ }^{29}$ Commentaire effectué par trois habitants d'Aubervilliers lors de leur participation à l'Atelier CAPADAPT organisée le 23 février 2019 à Aubervilliers.

30 Entretien avec un membre du groupement CARMA le 19 juillet 2018 à Argenteuil.
}

sionnement déjà en place au sein de la capitale (Macé Le Ficher, 2018). Qui plus est, élaborer de tels projets, ou s'en saisir, n'est pas accessible à tous. Les appels à projet deviendraient un outil de gentrification, avec l'exemple des jardins collectifs qui attirent une population éduquée, ayant les moyens de payer des loyers élevés (Ernwein et Tollis, 2017). Les appels à projets apparaissent dès lors plus comme une aide aux citoyens déjà organisés et mobilisés qu'à un moyen de réfléchir collectivement aux capabilités que l'ensemble de citoyens concernés a des raisons de valoriser.

La ville de Paris a lancé d'autres mesures afin de mieux intégrer les citoyens à une politique de développement durable: des comités de citoyens ont été créés en collaboration avec la ville, comme les Acteurs du Paris durable et les Volontaires pour le climat. Les acteurs administratifs ${ }^{31}$ souhaitent désormais, d'une part, dialoguer conjointement avec ces comités, ce qui initierait une désectorialisation des politiques climatiques $^{32}$. Ces mêmes acteurs veulent, d'autre part, réfléchir aux façons dont ces citoyens pourraient contribuer à la politique de la ville, ce qui favoriserait une meilleure intégration des initiatives citoyennes aux politiques publiques.

\section{Conclusion}

Les capabilités sont peu prises en compte actuellement dans les stratégies d'adaptation locales, qui apparaissent toujours secondaires par rapport à l'objectif de réduction des émissions. Toutefois, les différences entre territoires méritent d'être soulignées.

À Paris, les différents plans et appels à projets s'adressent principalement à une population déjà mobilisée, et déjà en capacité de s'en saisir. Afin de mobiliser une plus grande part de citoyens, une meilleure sensibilisation et une meilleure connaissance des impacts sont souvent proposées. Pourtant, le succès de l'appel à Volontaires pour le climat suggère que nombre de citoyens sont déjà sensibilisés à la cause. Pourquoi ces Parisiens souhaitent-ils s'engager? Comment pourraient-ils passer à l'action? Ce sont bien les questions de l'adoption de valeurs collectives et du renforcement des capabilités qui se posent.

Les administrations et élus de Plaine Commune et d'Aubervilliers ont le souci de donner au plus grand

\footnotetext{
31 Entretien avec un membre de l'Agence d'écologie urbaine, le 16 novembre 2018 à Paris; entretien avec un membre de la Mission Résilience de Paris, le 21 novembre 2018 à Paris.

32 La problématique n'est pas spécifique à la ville de Paris. Blanc et al. (2017), notamment, ont montré une forte sectorialisation des politiques appliquées à la végétation et au climat à l'échelle de la ville et de la région.
} 
nombre la possibilité de s'exprimer, et de faire le lien entre questions environnementales et de précarité. Toutefois, peu d'actions citoyennes sont proposées parmi les outils institutionnels par rapport à l'adaptation au changement climatique. La majorité des projets citoyens sont encore menés en parallèle de l'action publique au lieu de permettre un enrichissement mutuel.

À Gonesse, au sein de la communauté Roissy Pays de France, quelques associations environnementales sont très mobilisées. Mais pour éviter les tensions et protéger des projets d'aménagement contestés, les agents administratifs et les élus préfèrent s'appuyer sur des études techniques et consultent peu les citoyens.

Dans tous les cas, l'adoption de valeurs et de principes collectifs guidant la décision communale pourrait encourager les citoyens à se mobiliser pour le futur qu'ils souhaitent.

Si la participation publique aux différents plans envisageant l'adaptation à l'heure actuelle ne constitue pas un moyen de réfléchir aux capabilités citoyennes, ni à la façon dont elles pourraient être renforcées, la prise de conscience de la nécessité de mobiliser les citoyens à Paris pour atteindre l'objectif de neutralité carbone, de la nécessité de s'attaquer conjointement aux problèmes de précarité et environnementaux à Aubervilliers, ou de considérer ensemble développement local et protection de l'environnement à Gonesse, pourrait être favorable à une nouvelle approche de l'adaptation. Dans chacun de ces territoires, des citoyens sont conscients et mobilisés, mais ils ne trouvent pas encore de répondant institutionnel susceptible de valoriser leurs capabilités.

L'incapacité à valoriser les récits pour permettre de faire émerger les capabilités que les citoyens ont raison de valoriser est symptomatique des difficultés rencontrées par les démocraties représentatives à intégrer des procédures participatives. La pratique actuelle de l'adaptation met l'accent sur les diagnostics et les solutions technologiques qui nient les facteurs les plus fondamentaux de la vulnérabilité au changement climatique : l'accès faible et inéquitable aux ressources, aux services, à la prise de décisions et à la justice (Chandra et al., 2017). Certains auteurs pensent que les capacités d'adaptation au changement climatique sont un mélange de capacités génériques, liées au niveau d'éducation, d'accès à la ressource et aux soins de santé, ainsi que de capacités travaillées dans le cadre du changement climatique, par exemple, les connaissances relatives aux écosystèmes et à leurs fonctionnements (Eakin et al., 2014). Une position plus ouvertement politique postule que l'adaptation transformationnelle nécessite une redistribution du pouvoir au sein de la société (Blythe et al., 2018).

\section{Références}

Alkire S., Deneulin S., 2009. A normative framework for development, in Deneulin S. (Ed.), An introduction to the human development and capability approach, London, Earthscan, 25-35.

Antolinos-Basso D., Blanc N., Chiche J., Paddeu F., 2020. S'engager pour l'environnement dans le Grand Paris: territoires, politiques et inégalités, Cybergéo, https://doi. org/10.4000/cybergeo.34544.

Aubertin C., Damian M., Magny M., Millier C., Theys J., Treyer S., 2015. Introduction. Les enjeux de la conférence de Paris. Penser autrement la question climatique, Natures Sciences Sociétés, 23, S3-S5, https://doi.org/10.1051/nss/ 2015013.

Arnstein S.R., 1969. A ladder of citizen participation, JAIP, 35, 4, 216-224, https://doi.org/10.1080/01944366908977225.

Bacqué M.H., Biewener C. (Eds), 2014. L'empowerment, une pratique émancipatrice, Paris, La Découverte.

Bacqué M.H., Gauthier M., 2011. Participation, urbanisme et études urbaines. Quatre décennies de débats et d'expériences depuis «A ladder of citizen participation» de $\mathrm{S}$. R. Arnstein, Participations, 1, 36-66, https://doi.org/ 10.3917/parti.001.0036.

Ballet J., Bazin D., Dubois J., Mahieu F., 2011. A note on sustainability economics and the capability approach, Ecological Economics, 70, 11, 1831-1834, https://doi.org/ 10.1016/j.ecolecon.2011.05.009.

Ballet J., Bazin D., Pelenc J., 2015. Justice environnementale et approche par les capabilités, Revue de philosophie économique, 16, 13-39, https://doi.org/10.3917/ rpec.161.0013.

Basset T.J., Fogelman C., 2013. Déjà vu or something new? The adaptation concept in the climate change literature, Geoforum, 48, 42-53, https://doi.org/10.1016/j.geofo rum.2013.04.010.

Benitez F., Reghezza M., 2018. Les capabilités à faire face ou comment repenser la résilience des individus, VertigO, https://doi.org/10.4000/vertigo.19116.

Blanc N., Laigle L, 2018. Narratives, capabilities and climate change: towards a sustainable culture, in Birkeland I., Burton R., Parra C., Siivonen K. (Eds), Cultural sustainability and the nature-culture interface. Livelihoods, policies, and methodologies, New York, Routledge.

Blanc N., Glatron S., Lamarche T., Rankovic A., Sourdril A., 2017. Governance of urban nature, Articulo-Journal of Urban Research [Online], http://articulo.revues.org/3212.

Blondiaux L., Fourniau J.-M., 2011. Un bilan des recherches sur la participation du public en démocratie : beaucoup de bruit pour rien ?, Participations, 1, 1, 8-35, https://doi.org/ 10.3917/parti.001.0008.

Blythe J., Silver J., Evans L., Armitage D., Bennett N.J., Moore M.L., Morrison T.H., Brown K., 2018. The dark side of transformation: latent risks in contemporary sustainability discourse. Antipode, 50, 1206-1223, https://doi.org/ 10.1111/anti.12405. 
Brédif H., Bertrand F., Tabeaud M., 2015. Redéfinir le problème climatique par l'écoute du local: éléments de propédeutique, Natures Sciences Sociétés, 23, S65-S75, https://doi.org/10.1051/nss/2015019.

Cefaï D., 2010. Postface-L'expérience ethnographique, l'enquête et ses publics, in Cefaï D. (Ed.), L'engagement ethnographique, Paris, Éditions de l'EHESS, 547-598.

Chandra A., McNamara K.E., Dargusch P., 2017. The relevance of political ecology perspectives for smallholder climate-smart agriculture: a review. Journal of Political Ecology, 24, 821-842, https://doi.org/10.2458/v24i1.20969.

Chezel E., 2018. La fabrique collective des paysages climatiques - Une enquête avec les parcs éoliens citoyens en Frise $d u$ Nord. Thèse de doctorat en géographie, Grenoble, Université Grenoble Alpes.

Eakin H.C., Lemos M.C., Nelson D.R., 2014. Differentiating capacities as a means to sustainable climate change adaptation, Global Environmental Change, 27, 1-8, https://doi.org/10.1016/j.gloenvcha.2014.04.013.

Ernwein M., Tollis C., 2017. Produire la ville vivante: le travail des citadins et des non-humains, L'Information géographique, 81, 3, 13-31, https://doi.org/10.3917/ lig.813.0013.

Field C.B., Barros V.R., Dokken D.J., Mach K.J., Mastrandrea M.D., Bilir T.E, Chatterjee M., Ebi K.L, Estrada Y.O., Genova R.C, Girma B., Kissel E.S., Levy A.N, MacCracken S., Mastrandrea P.R., White L.L. (Eds), 2014. Changements climatiques 2014 : Incidences, adaptation et vulnérabilité - Résumé à l'intention des décideurs, Contribution du Groupe de travail II au cinquième Rapport d'évaluation du Groupe d'experts intergouvernemental sur l'évolution du climat, Genève, Organisation météorologique mondiale, https://archive.ipcc.ch/pdf/assessmentreport/ar5/wg2/ar5_wgII_spm_fr.pdf.

Flipo F., 2005. Pour une écologisation du concept de capabilité d'Amartya Sen, Natures Sciences Sociétés, 13, 1, 68-75, https://doi.org/10.1051/nss:2005010.

Godard O., 2010. Cette ambiguë adaptation au changement climatique, Nature Sciences Sociétés, 18, 3, 287-297, https://doi.org/10.1051/nss/2010036.
Lallau B., 2008. Les agriculteurs africains entre vulnérabilité et résilience. Pour une approche par les capabilités de la gestion des risques, Revue française de socio-économie, 1 , 177-198, https://doi.org/10.3917/rfse.001.0177.

Macé Le Ficher P., 2018. Appels à projets urbains innovants : l'alimentation au risque du food-washing?, Métropolitiques, www.metropolitiques.eu/Appels-a-projets-urbainsinnovants-l-alimentation-au-risque-du-food-washing.html.

Marquet V., Salles D., 2014. L'adaptation au changement climatique en France et au Québec. Constructions institutionnelles convergentes et diffusions contrastées, Critique Internationale, 62, 1, 73-91, https://doi.org/ 10.3917/crii.062.0073.

Nussbaum M., 2000. Women and human development-The capability approach, Cambridge, Cambridge University Press.

Pelling M., O’Brien K., Matyas D., 2014, Adaptation and transformation. Climatic Change, 133, 113-127, https://doi. org/10.1007/s10584-014-1303-0.

Pestre D., 2011. Des sciences, des techniques et de l'ordre démocratique et participative, Participations, 1, 210-238, https://doi.org/10.3917/parti.001.0210.

Rawls J., 2009. Théorie de la justice. Traduit de l'anglais par Catherine Audard, Paris, Le Seuil.

Ricoeur P., 1983. Temps et récit, Paris, Le Seuil.

Rousseau S., 2007. Vulnérabilité et résilience, analyse des entrées et sorties de la pauvreté : le cas de Manjakandriana à Madagascar, Mondes en développement, 140, 4, 25-44, https://doi.org/10.3917/med.140.0025.

Sen A., 1999. Development as freedom, Oxford, Oxford University Press.

Sen A., 2010. The idea of justice, London, Penguin books.

Simonet G., 2015. Une brève histoire de l'adaptation: l'évolution conceptuelle au fil des rapports du GIEC (1990-2014), Natures Sciences Sociétés, 23, S52-S64, https://doi.org/10.1051/nss/2015018.

Simonet G., 2016. De l'ajustement à la transformation : vers un essor de l'adaptation?, Développement durable \& territoires, 7, 2, 1-14, https://doi.org/10.4000/developpementdu rable. 11320 .

Citation de l'article : Pommerieux M., Blanc N., Laigle L., Tonnelat S. Capabilités et adaptation en Île-de-France. De la difficulté à intégrer les capabilités dans les plans locaux d'adaptation au changement climatique. Nat. Sci. Soc. 29, 2, 185-197. 